\title{
Partializing Stone Spaces using SFP domains ${ }^{\star}$ (Extended Abstract)
}

\author{
F. Alessi, P. Baldan, F. Honsell \\ Dipartimento di Matematica ed Informatica \\ via delle Scienze 208, 33100 Udine, Italy \\ \{alessi, baldan, honsell\}@dimi.uniud.it
}

\begin{abstract}
In this paper we investigate the problem of "partializing" Stone spaces by "Sequence of Finite Posets" (SFP) domains. More specifically, we introduce a suitable subcategory $\mathbf{S F P}^{m}$ of SFP which is naturally related to the special category of Stone spaces 2-Stone by the functor MAX, which associates to each object of SFP ${ }^{m}$ the space of its maximal elements. The category $\mathbf{S F} \mathbf{P}^{m}$ is closed under limits as well as many domain constructors, such as lifting, sum, product and Plotkin powerdomain. The functor MAX preserves limits and commutes with these constructors. Thus, SFP domains which "partialize" solutions of a vast class of domain equations in 2-Stone, can be obtained by solving the corresponding equations in $\mathbf{S F P} \mathbf{P}^{m}$. Furthermore, we compare two classical partializations of the space of Milner's Synchronization Trees using SFP domains (see [3], [15]). Using the notion of "rigid" embedding projection pair, we show that the two domains are not isomorphic, thus providing a negative answer to an open problem raised in [15].
\end{abstract}

\section{Introduction}

The problem of finding an appropriate "partialization" of a space of total elements, arises in several areas of Mathematics and Computer Science when dealing with computational approximations. A point can be taken as total if it can be separated from all the others points of the space by an intrinsic property. A "partialization" of a space of total elements can be viewed as a homeomorphic embedding of the space onto the maximal elements of a domain. Partial elements can then be seen as the representatives of possibly intensional properties of the original space.

Following the pioneering work of Scott, domains of approximations (essentially countably based continuous partial orders) have been used to study computability on real numbers and on other metric spaces (see e.g. $[19,14,10,12])$.

In this paper we investigate the "partialization" of 2-Stone spaces by SFP domains, first considered by Abramsky (see $[1,2,3]$ ). Both kinds of spaces play a fundamental rôle in the denotational semantics of concurrency. The importance of SFP domains is unquestionable (see [16]). The relevance of 2-Stone spaces, i.e.

\footnotetext{
* Partially supported by EC HCM project Lambda Calcul Typé, CHRX-CT92.0046.
} 
countably based, totally disconnected compact Hausdorff spaces, arises from the fact that compact ultrametric spaces, a category of spaces widely used in metric semantics (see [8]), are 2-Stone spaces.

A natural partialization of a 2-Stone space $\langle X, \tau\rangle$ by a Scott domain can be immediately obtained as the ideal completion of the collection $K \Omega_{n e}(X)$ of non-empty compact open subsets of $X$, ordered by reverse inclusion $D_{1}^{X}=$ Idl $\left(K \Omega_{n e}(X), \supseteq\right)$. Such domains are extensional in the sense that different partial elements approximate different sets of maximal elements. However, this class of domains is not closed under significant domain constructors, such as lifting and Plotkin powerdomain, in that such constructors add points that are meaningless w.r.t. the topology of the induced space.

Another extensional partialization can be obtained by associating to a 2Stone space $X$, the tree $D_{2}^{X}$ of closed balls of a metrization of $X$, ordered by reverse inclusion (as in $[6,7,12]$ ). In the setting of compact ultrametric spaces and non-distance increasing functions, domain constructors can be defined on these trees inducing the corresponding metric constructors on the space of maximal elements. This solution, however, is not completely satisfactory since the constructors are quite "ad hoc".

In this paper we explore the approach of [1] and consider, even non extensional, SFP domains. We exploit the fact that both 2-Stone spaces and SFP domains share the finitary property of being limits of sequences of finite discrete structures, namely finite discrete spaces and finite partial orders, respectively. In fact, at the level of finite structures, we have that:

i) partial orders are closed under many domain constructors, i.e. lifting $(.)_{\perp}$, separated sum + , product $\times$ and Plotkin powerdomain $\mathcal{P}_{P l}$;

ii) the subspace of maximal elements of a partial order is a discrete space, and every discrete space can be viewed as such a subspace, for suitable partial orders; iii) the natural functor MAX commutes in an obvious way with the domain constructors in i).

Thus, at the level of finite structures one can define compositionally natural partializations of discrete spaces. In this paper we generalize to the $\omega$-limit what happens at finite level. In particular we introduce a suitable subcategory $\mathbf{S F P}^{m}$ of $\mathbf{S F P} \mathbf{P}^{e p}$ closed under limits as well as the above mentioned domain constructors. The subspace of maximal elements of an object in $\mathbf{S F P}^{m}$ is a 2-Stone space, and every 2-Stone space can be viewed as such a subspace, for a suitable object in $\mathbf{S F P}^{m}$. Since the functor MAX from SFP $\mathbf{S P}^{m}$ to 2 -Stone is $\omega$-continuous, we can define SFP domains which "partialize" solutions of a vast class of domain equations in 2-Stone, by solving the corresponding equations in $\mathbf{S F P}^{m}$.

A partialization which has been extensively studied in the literature by Abramsky [3] and Mislove, Moss, Oles [15] is that of Milner's Synchronization Trees, or equivalently the closure of the space of hereditarily finite hypersets. This space is homeomorphic to the hyperuniverse $\mathcal{N}_{\omega}$ of [13] and it appears quite frequently under different mathematical perspectives, e.g. as the 2-Cantor space. In [15] the question was raised as to whether the two partializations given in [3] and [15] are isomorphic. 
An immediate application of our results shows that the solutions of the two domain equations in SFP ${ }^{e p}$ introduced by Abramsky (see [2]) and Mislove et al. (see [15]) have isomorphic maximal spaces. Using the notion of rigid embeddingprojection pair we give a negative answer to the open problem raised in [15]. The technique based on rigid embedding-projection pairs is rather promising in the analysis of the fine structure of domains. Using the above results, we can show furthermore that there is a plethora of non-isomorphic solutions of reflexive domain equations having the hyperuniverse $\mathcal{N}_{\omega}$ as space of total elements. It is a matter of further investigation which of these (if any) is the most appropriate partialization of the universe of hypersets.

Throughout the paper we use standard notation and basic facts of Domain Theory and Topology (see $[17,11]$ ). In Section 1 we give the basic definitions and we recall useful facts about SFP domains and Stone spaces. In Section 2 we discuss extensional partializations. In Section 3 we introduce the category SFP $^{m}$ and show that it is closed under various domain constructors. In Section 4 we relate 2-Stone spaces to $\mathrm{SFP}^{m}$ domains using the functor MAX. In Section 5 we discuss partialization of hyperuniverses. Finally in Section 6 we show that the results of sections 3-4 cannot be extended to function space constructors and that the compactness condition is necessary. For lack of space the proofs are omitted from this extended abstract. They appear in detail in [5].

This paper grew out from some initial results presented by the authors at the 1994 meeting in Rennes of the EEC project MASK (Mathematical Structures for Concurrency). The authors are grateful to S. Abramsky, P. Di Gianantonio, M. Lenisa and to all MASK members for useful comments.

\section{Stone Spaces and SFP Domains}

We start by recalling definitions and basic facts about Stone spaces and SFP's domains (see [17], [11] for more details). Both kinds of objects are finitary in the sense that they can be obtained as limits of sequences of finite objects in the corresponding categories.

Definition 1. A 2-Stone space is a compact topological space with a countable basis of clopen sets.

Proposition 2. Let $\langle X, \tau\rangle$ be a topological space. The following are equivalent: 1. $\langle X, \tau\rangle$ is a 2-Stone space;

2. $\langle X, \tau\rangle=\lim _{\leftarrow}\left\langle\left\langle X_{n}, \tau_{n}\right\rangle, f_{n}\right\rangle \quad\left(X_{n}\right.$ finite, $\tau_{n}$ discrete topology $)$;

3. $\langle X, \tau\rangle$ is compact and ultrametrizable with $d: X \times X \rightarrow\{0\} \cup\left\{2^{-n}\right\}_{n}$.

Let Top be the category of topological spaces and continuous functions. We denote with 2-Stone the full subcategory of Top consisting of 2-Stone spaces.

Given two cpo's $D$ and $E$, an embedding-projection pair (ep-pair) from $D$ to $E$ is any pair of continuous functions $i: D \rightarrow E, j: E \rightarrow D$ such that $i \circ j \sqsubseteq I d_{E}$ and $j \circ i=I d_{D}$. 
We denote by $\mathrm{CPO}^{e p}$ the category of CPO's and embedding-projection pairs. Let $\left\langle D_{n}, p_{n}\right\rangle$ be a sequence in $\mathbf{C P O}^{e p}$ and let $D$ be its limit. For all $n$ we denote with $i_{n}$ and $j_{n}$ the components of the ep-pair $p_{n}$ and with $\gamma_{n}=\left\langle\alpha_{n}, \beta_{n}\right\rangle$ the canonical ep-pair from $D_{n}$ into the limit.

Definition 3. A Sequence of Finite Posets (SFP) domain is a domain which is isomorphic to the direct limit of a directed sequence of finite CPO's in $\mathbf{C P O}^{e p}$.

We denote by $\mathbf{S F P}^{e p}$ the full subcategory of $\mathbf{C P O}^{e p}$ consisting of SFP's.

Let $X$ be a subset of the collection $K(D)$ of compact elements of $D$ and let $\mathcal{U}(X)$ denote the set of minimal upper bounds of $X . \mathcal{U}(X)$ is said to be complete if for each upper bound $y$ of $X$ there exists $x \in \mathcal{U}(X)$ such that $x \sqsubseteq y$. Finally $\mathcal{U}^{*}(X)$ denotes the smallest set containing $X$ and closed under $\mathcal{U}$.

Proposition 4. Let $(D, \sqsubseteq)$ be a partial order. Then $D$ is an SFP if and only if $D$ is an w-algebraic CPO and whenever $X$ is a finite set of finite elements of $D$, then $\mathcal{U}(X)$ is a complete finite set of upper bounds of $X$ and $\mathcal{U}^{*}(X)$ is finite.

If $D$ satisfies only the first two of the three conditions above it is called a $2 / 3$ $S F P$, or equivalently a coherent $\omega$-algebraic domain.

Proposition 5. Let $D=\lim _{\leftarrow}\left\langle D_{n}, p_{n}\right\rangle$ with $D_{n} S F P$ 's and $p_{n}$ ep-pairs. Then: 1. $u \subseteq_{f i n}(K(D)) \Leftrightarrow \quad \exists n . \exists u_{n} \subseteq_{f i n} K\left(D_{n}\right) u=\alpha_{n}\left(u_{n}\right)$;

2. $\forall n . \forall u_{n} \subseteq_{f i n} K\left(D_{n}\right) . \mathcal{U}^{*}\left(\alpha_{n}\left(u_{n}\right)\right)=\alpha_{n}\left(\mathcal{U}_{n}^{*}\left(u_{n}\right)\right)$.

\section{Extensional Partializations}

Given a 2-Stone space $\langle X, \tau\rangle$ we say that a SFP domain $D$ induces $\langle X, \tau\rangle$ if $(\operatorname{Max}(D), \mathcal{S}) \simeq\langle X, \tau\rangle$, where $\mathcal{S}$ denotes the topology induced by Scott topology on $\operatorname{Max}(D)$. In general, one can find infinitely many SFP domains which induce a given 2-Stone space $\langle X, \tau\rangle$; consider, for instance, all SFP's with a top element. The finite elements of any such domain, however, cannot be interpreted, in general, as the open sets (properties) of the original space. In order to have "partializations" of 2-Stone spaces where finite elements represent properties of the original space, it is natural to restrict attention to extensional domains.

Definition 6. An SFP domain $D$ is extensional if for each finite element $d \in D$

$$
d=\bigwedge\{z \mid z \in \operatorname{Max}(D) \cap \uparrow d\} .
$$

Notice that even if we restrict attention just to extensional SFP domains, still we cannot find a unique domain which induces a given 2-Stone space on its subspace of maximal elements. Consider, for instance, a flat domain and the meet-semilattice generated by it.

We discuss briefly two possible canonical constructions for embedding homeomorphically a 2 -Stone space $X$ into $\operatorname{Max}(D)$ for some domain $D$. 
The first construction is suggested by Stone duality [18] and it is obtained by considering the collection $K \Omega_{n e}(X)$ of non-empty compact open subsets of $X$, ordered by reverse inclusion $\left(K \Omega_{n e}(X), \supseteq\right)$ and then its ideal completion, $D_{1}^{X}=\operatorname{IdI}\left(K \Omega_{n e}(X), \supseteq\right)$. or equivalently the collection of non-empty compact subsets $\left(K_{n e}(X), \supseteq\right)$. Clearly $D_{1}^{X}$ is an extensional $\omega$-algebraic Scott domain and $\left(\operatorname{Max}\left(D_{1}^{X}\right), \mathcal{S}\right) \cong X$. Moreover $D_{1}^{X}$ is "maximal", in the sense that any other extensional SFP domain that induces $X$ can be embedded by a continuous injective function into $D_{1}^{X}$. In fact SFP domains are $\omega$-algebraic and each clopen is determined by a finite element. However, extensionality is not preserved by important domain constructors such as $\mathcal{P}_{P l}$. To see this it is enough to apply $\mathcal{P}_{P l}$ to the extensional finite SFP domain $D=\{a, b\}_{\perp}$.

Alternative extensional partializations are suggested by $[19,6,7,12]$. They are based on the fact that each 2-Stone space $X$ is metrizable with an ultrametric $d: X \times X \rightarrow\{0\} \cup\left\{2^{-n}\right\}_{n}$. Hence one can consider $D_{2}^{X}=\operatorname{Idl}\left(\left\{\bar{B}\left(x, 2^{-n}\right) \mid n \in\right.\right.$ $\mathbb{I N \}}, \supseteq) . D_{2}^{X}$ is an $\omega$-algebraic CPO where incomparable elements have no upper bounds, i.e. $D_{2}^{X}$ is a (finitely branching) tree. Maximal elements of $D_{2}^{X}$ can be identified with maximal chains in $\left(\left\{\bar{B}\left(x, 2^{-n}\right): n \in \mathbb{N}\right\}, \supseteq\right)$ and the function $f:\left(\operatorname{Max}\left(D_{2}^{X}\right), \mathcal{S}\right) \rightarrow(X, \Omega(X))$ mapping a chain $\left(B_{n}\right)_{n}$ to the sole point in $\bigcap_{n} B_{n}$ is a homeomorphism. This partialization contains only elements corresponding to a system of disjoint clopen sets. In $[6,7]$ it is shown that such trees (of formal balls), and level preserving functions, can be turned into a category BTree, which is equivalent to the cartesian closed category KUM of compact ultrametric spaces and non expansive functions. The equivalence is established by a functor that associates to each tree the space of maximal elements with the induced topology. In BTree we can define domain constructors, such as lifting, product, sum, function space and powerdomain, which induce on the space of maximal elements the corresponding metric constructors. This partialization is not completely satisfactory since it requires to restrict oneself to particular continuous functions (i.e. non expansive functions) and to consider constructors on trees which are quite "ad hoc".

\section{The Category SFP $^{m}$}

In view of the results of the previous section, in order to have a well behaved class of partializations, we are led to drop the extensionality condition and to focus on a wider class of SFP domains.

In this section we define a subcategory SFP $^{m}$ of SFP $\mathbf{P}^{e p}$ such that every object in $\mathbf{S F P}^{m}$ induces a 2-Stone space. Constructors over $\mathbf{S F P} \mathbf{P}^{m}$ are defined in the standard way. We establish a connection between these constructors and the corresponding ones over 2-Stone, using the functor Max. Then, a domain equation in 2-Stone can be translated into a domain equation in $\mathbf{S F P}^{m}$, in such a way that the solution of the latter is a partialization of the former.

We start by pointing out the "folklore" result that, if $D$ is a $2 / 3$ SFP then the Scott topology $\mathcal{S}$ and the Lawson topology $\mathcal{L}$ coincide on $\operatorname{Max}(D)$. Hence: 
Proposition 7 (Maximal elements of an SFP). Let $(D, \sqsubseteq)$ be a $2 / 3$ SFP. Then $(\operatorname{Max}(D), \mathcal{S})$ is a second countable, totally disconnected space.

Not all SFP domains induce a compact space on the subspace of maximal elements. Consider, for instance, $N_{\perp}$. A natural and sufficient, but not necessary, condition on $D$ for compactness to hold is that there exist a direct sequence with limit $D$, where projections preserve maximal elements. In order to single out a suitable category of such SFP domains (see Definition 12), we need some preliminary results.

Definition 8 (M-pair). Let $D$ and $E$ be SFP's. An ep-pair $p=\langle i, j\rangle: D \rightarrow E$ is called maximals preserving pair, or $M$-pair, if for all $x \in \operatorname{Max}(E), j(x) \in$ $\operatorname{Max}(D)$ (i.e. $j(\operatorname{Max}(E)) \subseteq \operatorname{Max}(D))$.

Notice that if $p=\langle i, j\rangle: D \rightarrow E$ is an M-pair then $j(\operatorname{Max}(E))=\operatorname{Max}(D)$. In fact, by surjectivity of $j$, for all $x \in \operatorname{Max}(D)$ there exists $y \in E$ such that $j(y)=x$. Hence if $y^{\prime} \in \operatorname{Max}(\uparrow y)$ we have $j\left(y^{\prime}\right)=x$. Moreover, composition of Mpairs is an M-pair. We denote by $\lim _{\rightarrow}^{m}\left\langle D_{n}, p_{n}\right\rangle$ the limit of a directed sequence of finite CPO's and M-pairs.

Lemma 9. Let $D=\lim _{\rightarrow}^{m}\left\langle D_{n}, p_{n}\right\rangle_{n}$. Then given $x=\left(x_{n}\right)_{n} \in D$ $x$ is maximal in $D$ iff $j_{n}(x)=x_{n}$ is maximal in $D_{n}$ for all $n$.

Continuity in Lawson topology is a stronger notion than continuity in Scott topology, but one can easily check that projections are also Lawson continuous. This simple remark is useful in proving the following:

Lemma 10. Let $D=\lim _{\rightarrow}^{m}\left\langle D_{n}, p_{n}\right\rangle_{n}$. Then $\operatorname{Max}(D)$ is Lawson closed, hence compact.

Theorem 11. Let $D=\lim _{\rightarrow}^{m}\left\langle D_{n}, p_{n}\right\rangle_{n}$. Then $(\operatorname{Max}(D), \mathcal{S})$ is a 2-Stone space.

Finally we can introduce the category of SFP domains we shall work with:

Definition 12 (Category SFP ${ }^{m}$ ). The category $\mathbf{S F P}^{m}$ has as objects those SFP's that are limit of directed sequences of finite CPO's and M-pairs. Morphisms are M-pairs, the identity and composition are standard.

We can give also an intrinsic characterization of $\mathbf{S F P}^{m}$ objects. This will be instrumental in proving some interesting properties of $\mathbf{S F P}^{m}$ such as the closure with respect to direct limits.

Definition 13 (M-condition). We say that an $\operatorname{SFP}(D$, $)$ satisfies the $M$ condition if $\forall u \subseteq_{f i n} K(D) . \exists v \subseteq_{f i n} K(D)$ such that:

i) $u \subseteq v$,

ii) $\operatorname{Max}\left(\mathcal{U}^{*}(v)\right) \sqsubseteq_{s} \operatorname{Max}(D)$, where $\sqsubseteq_{s}$ is Smyth preorder (i.e. $u \sqsubseteq_{s} v$ iff $\forall y \in$ $v . \exists x \in u . x \sqsubseteq y$ ). 
In order to show that $\mathbf{S F P}^{m}$ objects are exactly those SFP's which satisfy the M-condition we proceed as follows. First we prove that the limit, taken in $\mathbf{S F P}^{e p}$, of a sequence $\left\langle D_{n}, p_{n}\right\rangle$ in $\mathbf{S F P} \mathbf{P}^{m}$ is a limit in $\mathbf{S F P} \mathbf{P}^{m}$. Then we show that the M-condition is preserved under limits. Using these facts and that every finite CPO satisfies the M-condition, we can easily prove the desired result.

Lemma 14. Let $D=\lim _{\rightarrow}\left\langle D_{n}, p_{n}\right\rangle$, with $\left\langle D_{n}, p_{n}\right\rangle$ directed sequence in $\mathbf{S F P}^{m}$. Then $\quad x=\left(x_{n}\right)_{n} \in \operatorname{Max}(D)$ iff $x_{n} \in \operatorname{Max}\left(D_{n}\right)$ for all $n$.

Lemma 15. Let $D=\lim _{\rightarrow}\left\langle D_{n}, p_{n}\right\rangle$, with $\left\langle D_{n}, p_{n}\right\rangle$ directed sequence in $\mathbf{S F P}$. If each $D_{n}$ satisfies the $M$-condition then also $D$ satisfies $M$-condition.

Theorem 16 (Internal characterization of SFP ${ }^{m}$ objects). Let (D, ㄷ) be an SFP. Then $D$ is an $\mathbf{S F P}^{m}$ object iff $D$ satisfies the $M$-condition.

Corollary 17. The category $\mathrm{SFP}^{m}$ is closed under direct limits.

Notice that given a 2-Stone space $X$ the domains $D_{1}^{X}$ and $D_{2}^{X}$ defined in section 1 are both SFP objects which satisfy the the M-condition. As we mentioned earlier, however, the category SFP $^{m}$ does not contain all SFP's that induce 2-Stone spaces, i.e. the M-condition is only sufficient, but not necessary for the compactness of the induced space. Consider for instance the functor $+^{*}$ over SFP ${ }^{e p}$ defined as follows:

$D+^{*} E={ }^{\operatorname{def}}\left(\{(d, 0) \mid d \in D\} \cup\{(e, 1) \mid e \in E\} \cup\{\perp, *\}, \sqsubseteq^{*}\right)$, where for each $x, y \neq *, x \sqsubseteq^{*} y$ if and only if $x \sqsubseteq_{D+E} y$ and $\left(\perp_{D}, 0\right) \sqsubseteq^{*} *,\left(\perp_{E}, 1\right) \sqsubseteq^{*} *$.

Given two strict functions $f: D \rightarrow D^{\prime}, g: E \rightarrow E^{\prime}, f+^{*} g$ coincides with $f+g$ on all the elements different from $*$ and it maps $*_{D+{ }^{*} E}$ to $*_{D^{\prime}+{ }^{*} E^{\prime}}$. The action of $+^{*}$ over M-pairs is $\langle i, j\rangle+{ }^{*}\langle h, k\rangle=\left\langle i+{ }^{*} h, j+{ }^{*} k\right\rangle$.

It is easy to prove that the initial solution of the domain equation $X \simeq X+{ }^{*} X$ is not in $\mathbf{S F P}^{m}$ but that the space of its maximal elements is 2-Stone.

We show now that several domain constructors over SFP ${ }^{e p}$, namely lifting $(.)_{\perp}$, separated sum + , product $\times$ and Plotkin powerdomain $\mathcal{P}_{P l}$, are functorial over $\mathbf{S F P} \mathbf{P}^{m}$. The coalesced sum $\ominus$ is functorial only on $\mathbf{S F P}_{0}^{m}$, the subcategory of SFP $^{m}$ consisting of non-trivial SFP domains. From now on it will be understood that the application of the $\oplus$ functor is confined to (objects in) $\mathbf{S F P}_{0}^{m}$. The function space constructor is very problematic, see Section 6 for a brief discussion of this issue.

We shall use the characterization of Plotkin powerdomain $\mathcal{P}_{P t}(D)$ as the set $\{X \subseteq D \mid X$ non-empty, convex and Lawson closed $\}$, with the Egli-Milner ordering. $\operatorname{Con}(X)$ denotes the least convex set that contains $X . C l$ denotes the closure operator in Lawson topology. If $f: D \rightarrow E$ is a continuous function then $\mathcal{P}_{P l}(f): \mathcal{P}_{P l}(D) \rightarrow \mathcal{P}_{P l}(E)$ is defined as $\mathcal{P}_{P l}(f)(X)=\operatorname{Con}(C l(f(X)))$. In particular if $f$ is a projection then $\mathcal{P}_{P l}(f)(X)=f(X)$. In fact a projection is Lawson continuous, hence $f(X)$ is closed. Moreover $f(X)$ is convex.

The next lemma gives a characterization of $\operatorname{Max}\left(\mathcal{P}_{P l}(D)\right)$ for an $\mathbf{S F P}^{m}$ object $D$. It states that only maximal elements of $D$ play an essential role in forming maximal elements of the Plotkin powerdomain. It will be used to show that $\mathcal{P}_{P l}$ 
is functorial on $\mathbf{S F P}^{m}$ and corresponds, in a sense formalized in Section 4 to the constructor $\mathcal{P}_{n c o}$ of 2-Stone.

Lemma 18. Let $D$ be an $\mathbf{S F P}^{m}$ object. Then $\operatorname{Max}\left(\mathcal{P}_{P l}(D)\right)=\left\{X \in \mathcal{P}_{P l}(D) \mid\right.$ $X \subseteq \operatorname{Max}(D)\}$.

Since each subset of $\operatorname{Max}(D)$ is clearly convex we have $\operatorname{Max}\left(\mathcal{P}_{P l}(D)\right)=\{X \subseteq$ $\operatorname{Max}(D) \mid X$ Lawson closed $\}$.

Lemma 19. Let $D, E, D_{i}, E_{i}(i=1,2)$ be $\mathbf{S F P}^{m}$ objects and let $p: D \rightarrow E$, $p_{i}: D_{i} \rightarrow E_{i}$ be M-pairs. Then:

1. $p_{\perp}: D_{\perp} \rightarrow E_{\perp}$;

2. $p_{1}+p_{2}: D_{1}+D_{2} \rightarrow E_{1}+E_{2}$;

3. $p_{1} \oplus p_{2}: D_{1} \oplus D_{2} \rightarrow E_{1} \oplus E_{2} \quad$ (if $\left.\left|D_{1}\right|,\left|D_{2}\right|>1\right)$;

4. $p_{1} \times p_{2}: D_{1} \times D_{2} \rightarrow E_{1} \times E_{2}$;

5. $\mathcal{P}_{P l}(p): \mathcal{P}_{P l}(D) \rightarrow \mathcal{P}_{P l}(E)$ are M-pairs.

Notice that if $D_{1}$ or $D_{2}$ is a one-point CPO then $p_{1} \oplus p_{2}$ can fail to be an M-pair. Hence, as remarked, $\oplus$ is not functorial on $\mathbf{S F P}^{m}$.

Closure of $\mathbf{S F P}^{m}$ with respect to all constructors defined above easily follows from a general result.

Lemma 20. Let $F:\left(\mathbf{S F P}^{e p}\right)^{n} \rightarrow \mathbf{S F P}^{e p}$ be a locally continuous functor that preserves $M$-pairs. If $D_{1}, \ldots, D_{n}$ are SFP $^{m}$ objects then $F\left(D_{1}, \ldots, D_{n}\right)$ is an SFP ${ }^{m}$ object.

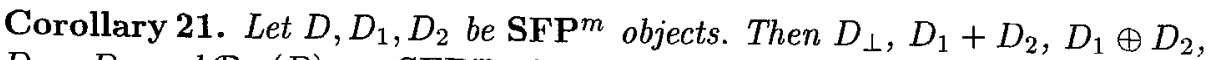
$D_{1} \times D_{2}$ and $\mathcal{P}_{P l}(D)$ are $\mathbf{S F P}^{m}$ objects.

Corollary 22 (Domain constructors in SFP ${ }^{m}$ ). The constructors $(.)_{\perp},+$, $\times$ and $\mathcal{P}_{P l}$ are functorial over $\mathbf{S F P}^{m}$. The constuctor $\oplus$ is functorial over the category SFP $_{0}^{m}$.

\section{Relation between SFP ${ }^{m}$ and 2-Stone}

In this section we relate the categories $\mathbf{S F P}^{m}$ and 2-Stone. First of all we show that it is possible to define an $\omega$-continuous functor MAX: $\mathbf{S F P}^{m} \rightarrow 2$-Stone. Then we prove that the functor MAX is compositional with respect to the constructors considered in the previous section, in the sense that $\operatorname{MAX}(F(D)) \simeq$ $\bar{F}(\operatorname{MAX}(D))$, where $\bar{F}$ is the functor over 2-Stone corresponding to $F$. In this way equations in 2-Stone and their solutions can be described by means of equations and solutions in $\mathbf{S F P}^{m}$.

Definition 23. The contravariant functor MAX: SFP $\rightarrow$ 2-Stone is defined as follows. For each $\mathbf{S F P}^{m}$ object $D, \operatorname{MAX}(D)=(\operatorname{Max}(D), \mathcal{S})$. For each M-pair $p=\langle i, j\rangle: D \rightarrow E, \operatorname{MAX}(p)=j_{\mid \operatorname{Max}(E)}: \operatorname{MAX}(E) \rightarrow \operatorname{MAX}(D)$. 
It is straightforward to check that MAX is well-defined and $\omega$-continuous:

Theorem 24. Let $D=\lim _{\rightarrow}\left\langle D_{n}, p_{n}\right\rangle$, with $\left\langle D_{n}, p_{n}\right\rangle$ a direct sequence in $\mathbf{S F P}^{m}$, or $\operatorname{SFP}_{0}^{m}$. Then $\operatorname{MAX}(D) \simeq \lim _{\leftarrow}\left\langle\operatorname{MAX}\left(D_{n}\right), \operatorname{MAX}\left(j_{n}\right)\right\rangle$.

The correspondence between constructors in $\mathbf{S F P}^{m}$ and in 2-Stone is formalized as follows:

Definition 25. Two functors $F:(\text { 2-Stone })^{n} \rightarrow$ 2-Stone and $G:\left(\mathbf{S F P}^{m}\right)^{n} \rightarrow$ SFP $^{m}$ are called associated functors if there exists a natural isomorphism $\eta$ : $F \circ(\operatorname{MAX}, \ldots, \operatorname{MAX}) \rightarrow$ MAX。G.

We now show that $(.)_{\perp},+, \times$ and $\mathcal{P}_{P l}$ in $\mathbf{S F P}^{m}$ are associated to the corresponding constructors $I d$ (identity), $\cup$ (disjoint union), $\times$ (product), and $\mathcal{P}_{n c o}$ (hyperspace of non-empty compact subsets) in 2-Stone. ${ }^{2}$ Moreover, in $\mathbf{S F P}_{0}^{m}$, the constructor $\oplus$ is associated to $\bar{U}$.

Lemma 26. Let $D, D_{1}$ and $D_{2}$ be $\mathbf{S F P}^{m}$ objects. Then

1. $\operatorname{MAX}\left(D_{1}+D_{2}\right) \simeq \operatorname{MAX}\left(D_{1}\right) \operatorname{UMAX}\left(D_{2}\right)$;

2. $\operatorname{MAX}\left(D_{\perp}\right) \simeq \operatorname{MAX}(D)$

3. $\operatorname{MAX}\left(D_{1} \times D_{2}\right) \simeq \operatorname{MAX}\left(D_{1}\right) \times \operatorname{MAX}\left(D_{2}\right)$;

4. $\operatorname{MAX}\left(\mathcal{P}_{P l}(D)\right) \simeq \mathcal{P}_{\text {nco }}(\operatorname{MAX}(D))$;

5. $\operatorname{MAX}\left(D_{1} \oplus D_{2}\right) \simeq \operatorname{MAX}\left(D_{1}\right) \cup \operatorname{MAX}\left(D_{2}\right)$.

Theorem 27. The following functors on $\mathbf{S F P}^{m}$ and 2-Stone are associated: $(.)_{\perp}$ with $I d, \times$ with $\times,+$ with $\bigcup$ and $\mathcal{P}_{P l}$ with $\mathcal{P}_{\text {nco }}$. Moreover $\oplus$ over $\mathbf{S F P}_{0}^{m}$ is associated to $\cup$ over 2-Stone. Finally composition of associated functors is the functor associated to the composition.

\section{Domain Equations for Non Well Founded Sets}

In this section we apply the theory developed in the previous section to the study of the initial solutions of two important domain equations in $\mathbf{S F P}^{m}$, namely:

$$
\begin{aligned}
& X \simeq\left(2 \oplus \mathcal{P}_{P l}\left(X_{\perp}\right)\right) \\
& X \simeq 1+\mathcal{P}_{p l}(X)
\end{aligned}
$$

The initial solution $\bar{D}$ of (Eq1) was introduced by Abramsky in [3] in order to partialize Milner's Synchronization Trees. The initial solution $\bar{E}$ of $(E q 2)$ was introduced by Mislove Moss and Oles in [15] in order to partialize the closure of the space of hereditarily finite hypersets. This space of hypersets is homeomorphic in 2-Stone to Milner's Synchronization Trees, as can be seen, for instance, by an immediate application of Theorem 27 and Theorem 24. In [15] the question was raised as to whether the two initial solutions in SFP are isomorphic.

${ }^{2}$ The space $\mathcal{P}_{n c o}(X)$ is defined as the set $\{K \subseteq X \mid K$ non-empty and compact $\}$ endowed with the Vietoris topology, i.e. the topology having as subbasis: $\mathcal{V}_{A}=$ $\left\{K \in \mathcal{P}_{n c o}(X) \mid K \subseteq A\right\}$ and $\mathcal{Z}_{A}=\left\{K \in \mathcal{P}_{n c o}(X) \mid K \cap A \neq \emptyset\right\}$ for $A \in \Omega(X)$. 
We give a negative answer to this open problem by showing that $\bar{D}$ and $\bar{E}$ are non-isomorphic. Our proof is based on the notion of rigid ep-pair.

Using the above results, we can show furthermore that there is a plethora of non-isomorphic solutions of reflexive domain equations having the hyperuniverse $\mathcal{N}_{\omega}$ as space of total elements. In general, for any SFP domain $D_{0}$ such that $U=\operatorname{MAX}\left(D_{0}\right)$ is a finite discrete space, the initial solutions of the equations $X \simeq\left(D_{0}+\mathcal{P}_{P l}(X)\right)$ and (if $D_{0}$ has at least two points) $X \simeq\left(D_{0} \oplus \mathcal{P}_{P l}\left(X_{\perp}\right)\right)$ induce the hyperuniverse $\mathcal{N}_{\omega}(U)$ ([13]).

The proof of the fact that $D$ and $E$ are not isomorphic is done through an analysis of the fine structure of Plotkin powerdomain constructor. This allows to show that $D$ contains some points in a particular relation with the maximal elements of $D$ which do not exist in $E$.

We work in SFP ${ }^{e p}$. First we introduce the notion of rigid ep-pair and list some of its most important properties:

Definition 28 (Rigid ep-pair). Let $D$ and $E$ be SFP's. An ep-pair $p=\langle i, j\rangle$ : $D \rightarrow E$ is called rigid if $\forall x \in D$ and $y \in E$ with $y \sqsubseteq i(x)$, there exists $x^{\prime} \in D$ such that $x^{\prime} \sqsubseteq x$ and $i\left(x^{\prime}\right)=y$.

Proposition 29. Let $D$ and $E$ be SFP's and let $p=\langle i, j\rangle: D \rightarrow E$ be an eppair. Then the following statements are equivalent:

1. $p$ is rigid;

2. for all $x \in D$ and $y \in E$, if $y \sqsubseteq i(x)$ then $i \circ j(y)=y$;

3. for all $x, x^{\prime} \in D, y \in E$ with $i(x) \sqsubseteq y \sqsubseteq i\left(x^{\prime}\right)$, there exists $x^{\prime \prime} \in D$ such that $x \sqsubseteq x^{\prime \prime} \sqsubseteq x^{\prime}$ and $y=i\left(x^{\prime \prime}\right)$.

Lemma 30. Composition of rigid ep-pairs is a rigid ep-pair.

Lemma 31. Let $D, D^{\prime}, E, E^{\prime}$ be SFP's and let $p=\langle i, j\rangle: D \rightarrow E, p^{\prime}=\left\langle i^{\prime}, j^{\prime}\right\rangle$ : $D^{\prime} \rightarrow E^{\prime}$ be rigid ep-pairs. Then

1. $p_{\perp}: D_{\perp} \rightarrow E_{\perp}$

2. $p \times p^{\prime}: D \times D^{\prime} \rightarrow E \times E^{\prime}$,

3. $p+p^{\prime}: D+D^{\prime} \rightarrow E+E^{\prime}$,

4. $p \oplus p^{\prime}: D \oplus D^{\prime} \rightarrow E \oplus E^{\prime}$,

5. $\mathcal{P}_{p l}(p): \mathcal{P}_{p l}(D) \rightarrow \mathcal{P}_{p l}(E)$

are rigid ep-pairs.

Lemma 32. Let $\left\langle D_{n}, p_{n}\right\rangle$ be a directed sequence of SFP's and ep-pairs. Let $D=$ $\lim _{\rightarrow} D_{n}$ be the direct limit of the sequence. If every $p_{n}$ is rigid then the canonical ep-pairs $\left\langle\alpha_{n}, \beta_{n}\right\rangle: D_{n} \rightarrow D$ are rigid.

Finally we are able to state the property satisfied by $\bar{D}$ but not by $\bar{E}$. The two results below are proved using essentially the fact that both $\bar{D}$ and $\bar{E}$ are limits of sequences with rigid ep-pairs. Hence the property is shown to hold (fail) in the limit by testing it at each finite level.

Lemma 33. There exists $a, b \in K(\bar{D})$, with $a \sqsubset b$ such that 1. $\forall x \in \bar{D} . a \sqsubseteq x \sqsubseteq b \quad \Rightarrow \quad x=a \vee x=b$, 
2. $\forall x \in \bar{D} . \perp \sqsubseteq x \sqsubseteq a \Rightarrow x=\perp \vee x=a$,

3. $\operatorname{Max}(\uparrow a)=\operatorname{Max}(\uparrow b)$.

Lemma 34. There are no elements $a, b \in K(\bar{E})$, with $a \sqsubset b$ such that

1. $\forall x \in \bar{E} . a \sqsubseteq x \sqsubseteq b \quad \Rightarrow \quad x=a \vee x=b$;

2. $\forall x \in \bar{E} . \perp \sqsubseteq x \sqsubseteq a \Rightarrow x=\perp \vee x=a$;

3. $\operatorname{Max}(\uparrow a)=\operatorname{Max}(\uparrow b)$.

Theorem 35. The initial solutions of (Eq1) and (Eq2) are not isomorphic.

\section{Final remarks}

1. Given an SFP domain $D$, the space $\operatorname{MAX}(D)$ is a space with a countable basis of clopen sets. One can ask whether Theorem 27 can be extended to SFP ${ }^{e p}$ and QStone, the category of totally disconnected separable Hausdorff spaces and continuous functions. The answer is negative, since there is no associated functor to Plotkin powerdomain constructor when we drop the compactness condition. Let $D_{1}=N_{\perp}, D_{2}=N_{\perp}+\mathbb{N}_{\perp}$. Both $\operatorname{Max}\left(D_{1}\right)$ and $\operatorname{Max}\left(D_{2}\right)$ coincide with $\mathbb{N}$ endowed with the discrete topology. But $\operatorname{Max}\left(\mathcal{P}_{p l}\left(D_{1}\right)\right)$ is not homeomorphic to $\operatorname{Max}\left(\mathcal{P}_{p l}\left(D_{2}\right)\right)$ since the former has only one limit point, while the latter has more than one. In fact, in $\operatorname{Max}\left(\mathcal{P}_{p l}\left(D_{1}\right)\right)$ there is a unique infinite set, namely $D_{1}$ itself, while $\operatorname{Max}\left(\mathcal{P}_{p l}\left(D_{2}\right)\right)$ contains more than one infinite element.

2. It would be interesting to extend the results of Section 4 so as to comprise also the function space constructor. Unfortunately 2-Stone is not cartesian closed, in that the space of continuous functions between two 2-Stone spaces endowed with the compact open topology is not compact, in general. One could then try to look at least for the existence of some functor over 2-Stone associated to the function space constructor over SFP. But even this is hopeless.

First of all maximal functions between SFP objects do not map maximal elements into maximal elements, and thus they do not induce in a natural way functions between the spaces of maximal points. Consider, for instance, $D=$ $N_{\text {lazy }}, B o o l=\{t t, f f\}_{\perp}$ and take the continuous function parity $: D \rightarrow$ Bool (defined in the obvious way). It is a maximal element in $[D \rightarrow B o o l]$, but it does not map the maximal point $\omega \in D$ in a maximal element of Bool.

But furthermore, function spaces of SFP objects, with the same space of maximal elements, can be non-homeomorphic. Consider, for instance,

$$
E=\{a, b, \perp\} \cup\left\{c_{i} \mid i \in N\right\},
$$

ordered as follows: for all $i, c_{i} \sqsubseteq a, b$, and for all $x, \perp \sqsubseteq x$.

Then $\operatorname{Max}(B o o l)$ and $\operatorname{Max}(E)$ are the same discrete space, but the maximal elements of the function spaces $\operatorname{Max}([$ Bool $\rightarrow$ Bool $])$ and $\operatorname{Max}([$ Bool $\rightarrow E])$ are different. In fact $\operatorname{Max}([B o o l \rightarrow B o o l])$ is a finite discrete space containing only four functions, while $\operatorname{Max}([\mathrm{Bool} \rightarrow E])$ contains infinitely many functions. Namely, the functions $f_{i}(t t)=a, f_{i}(f f)=b, f_{i}(\perp)=c_{i}$, for $i \in \mathbb{N}$, and the constant functions. All these functions are isolated points in a topological sense 
(since they are finite elements in the SFP) and thus $\operatorname{Max}([$ Bool $\rightarrow E])$ is a infinite discrete space and hence it is not compact. This latter example shows also that SFP $^{m}$ is not closed w.r.t the function space constructor.

\section{References}

[1] Samson Abramsky. Total vs. partial object and fixed points of functors. Unpublished Manuscript, 1985.

[2] Samson Abramsky. A Cook's tour of the finitary non-well-founded sets. Talk delivered at BTCS Colloquium, 1988.

[3] Samson Abramsky. A domain equation for bisimulation. Information and Computation, 92(2):161-218, 1991.

[4] Samson Abramsky. Domain theory in logical form. Annals of Pure and Applied Logic, 51:1-77, 1991.

[5] F. Alessi, P. Baldan and F. Honsell. Partializing Stone Spaces using SFP domains. Technical Report. University of Udine.

[6] F. Alessi and M. Lenisa. Stone duality for trees of balls. Talk delivered at MASK workshop, Koblenz, 1993.

[7] P. Baldan. A fixed point theorem for the solution of domain equations in a category of trees. Tesi di Laurea, Udine 1994.

[8] J.W. de Bakker and E. de Vink. Control Flow Semantics. MIT Press, 1996.

[9] J.W. de Bakker and J.I. Zucker. Processes and the denotational semantics of concurrency. Information and Control, 54(1/2):70-120, 1982.

[10] P. Di Gianantonio. Real number computability and domain theory. Information and Computation, 127(1):11-25, 1996.

[11] J. Dugundji. Topology. Allyn and Bacon, 1966.

[12] A. Edalat and R. Heckmann. A computational model for metric spaces. 1996, to appear.

[13] M. Forti, F. Honsell, and M. Lenisa. Processes and hyperuniverses. MFCS '93, LNCS 841:352-367, 1994.

[14] M.E. Majster-Cederbaum and F. Zetzsche. Towards a foundations for semantics in complete metric spaces. Information and Computation, 90:217-243, 1991.

[15] M.W. Mislove, L.S. Moss, and F.J. Oles. Non-well-founded sets modeled as ideal fixed points. Information and Computation, 93(1):16-54, 1991.

[16] Gordon D. Plotkin. A powerdomain construction. SIAM Journal on Computing, 5(3):452-487, 1976.

[17] Gordon D. Plotkin. Domains. Unpublished Course Notes. University of Edinburgh, 1983.

[18] Marshall H. Stone. The theory of representations for Boolean algebras. Transactions of the American Mathematical Society, 40:37-111, 1936.

[19] K Weihrauch and U. Shreiber. Embedding metric spaces into cpo's. Theoretical Computer Science, 16(1):5-24, 1981. 